\title{
Identificación de problemas y propuestas para mejorar la atención de las urgencias extrahospitalarias en Navarra: un estudio Delphi
}

\author{
Identification of problems and proposals for improving \\ outpatient emergency care in Navarre: a Delphi study
}

\author{
I. Pérez-Ciordia' ${ }^{1}$ A. Brugos ${ }^{1}$, F. Guillén-Grima ${ }^{2}$, C. Fernández-Martínez ${ }^{1}$, I. Aguinaga²
}

\section{RESUMEN}

Fundamento. Las urgencias extrahospitalarias de Navarra sufrieron modificaciones en la organización hace dos años, destacando la creación del servicio de urgencias rural (SUR) que coexiste geográficamente con el servicio normal de urgencias. El objetivo del estudio es conocer la opinión de expertos sobre los problemas y las recomendaciones de mejora que afectan a las urgencias extrahospitalarias.

Metodología. Se realizó un estudio Delphi con la participación de 37 expertos (médicos y enfermeras). Por medio del correo electrónico se realizan 3 rondas de cuestionarios sucesivos. El último cuestionario identifica el grado de acuerdo con las ideas del grupo y se identifican los 15 problemas y recomendaciones más relevantes, seleccionando aquellas ideas que alcancen, al menos, el $50 \%$ de acuerdo y una prioridad superior al percentil 25. Agrupamos las ideas mediante análisis de clúster jerárquico.

Resultados. Se identifican 4 clúster de problemas, destacando entre ellos que "la población utiliza las urgencias para patología banal, como una consulta más" con una puntuación de 297 puntos. De las recomendaciones, con 3 clúster identificados, destaca la "necesidad de diseñar un plan estratégico de atención a las urgencias con unos recursos acorde a las necesidades" (310 puntos de prioridad) y "enviar mensajes claros a la población sobre uso correcto de urgencias" (192 puntos de prioridad).

Conclusión. El método Delphi identifica problemas y áreas de mejora a través del consenso.

Palabras clave. Delphi. Urgencias. Servicios de Salud.

\begin{abstract}
Background. Two years ago there were alterations to outpatient A and E departments in Navarre; in particular, a rural A and E service (Servicio de Urgencias Rural, SUR) was set up, which coexists geographically with the normal A and E service. The aim of this study is to determine the opinion of experts on problems and recommendations for improvements that affect outpatient $\mathrm{A}$ and $\mathrm{E}$ services.
\end{abstract}

Methodology. A Delphi study was carried out with the participation of 37 experts (doctors and nurses). Three successive rounds of questionnaires were completed using email. The final questionnaire identified degree of agreement with the group's ideas, and the 15 most relevant problems and recommendations were identified; those ideas that were selected achieved at least $50 \%$ agreement and their priority was above $25 \%$. We grouped the ideas using a hierarchic cluster analysis.

Results. Four cluster problems were identified; the most outstanding amongst them being "the population uses $\mathrm{A}$ and $\mathrm{E}$ for banal pathologies, just like any other consultation" with a score of 297 points. Outstanding amongst the recommendations, with 3 identified clusters, were the "need to design a strategic plan for A and E care with resources appropriate to the needs" (310 priority points) and "sending clear messages to the population on the correct use of A and E" (192 priority points).

Conclusion. The Delphi method identifies problems and improvement areas through consensus.

Key words. Delphi. Emergency. Health service.

An. Sist. Sanit. Navar. 2011; 34 (3): 395-407

1. Servicio Navarro de Salud-Osasunbidea. Pamplona.

2. Universidad Pública de Navarra. Pamplona.

Recepción: 23 de mayo de 2011

Aceptación provisional: 11 de julio de 2011

Aceptado definitiva: 14 de septiembre de 2011

\section{Correspondencia:}

Ignacio Pérez Ciordia

Servicio de Urgencias de Tafalla

Subdirección de Urgencias extrahospitalarias

Pza. de la Paz, s/n

31002 Pamplona

E-mail: ignacio_perez_ciordia@hotmail.com 


\section{INTRODUCCIÓN}

La demanda de asistencia sanitaria urgente presenta en los últimos años un crecimiento descontrolado, que provoca crecientes dificultades para una provisión de servicios de calidad $^{1-4}$. Existe discrepancia sobre lo que se considera un uso adecuado de los servicios de urgencia, tanto desde la perspectiva del paciente como de los profesionales sanitarios ${ }^{5,6}$.

Se ha constatado que el uso de las urgencias para patología banal tiene importantes repercusiones negativas en tiempos de espera, coste y disminución de la calidad ${ }^{7-9}$.

Varios factores se han asociado al uso inadecuado de urgencias, tanto sanitarios como extrasanitarios, con importantes repercusiones en su gestión. Se asocian a una mayor frecuentación el nivel socioeconómico bajo $^{10}$, la mayor accesibilidad ${ }^{7,11}, \mathrm{y}$ la edad menor de un año ${ }^{6,12}$. La población inmigrante presenta una frecuentación similar a la española ${ }^{13}$.

Se han ensayado diferentes medidas para minimizar este problema entre las que se destacan las intervenciones educativas poblacionales $^{14-17}$, medidas de copago $^{18,19}$, derivación a otros dispositivos asistencia$\operatorname{les}^{20,21}$, hospitalización a domicilio ${ }^{22}$, aumento de recursos en la atención ${ }^{11}$, etc. Las rescisiones señalan que las intervenciones son de escasa efectividad ${ }^{22}$. En general se acepta la necesidad de incorporar una mejor coordinación y gestión de los servicios así como más recursos financieros ${ }^{2,23,24}$.

Las nuevas tecnologías de comunicación e información posibilitan nuevos enfoques organizativos que pueden permitir mejoras en el funcionamiento de los diferentes dispositivos asistenciales ${ }^{25}$.

En Navarra, desde el año 2008 y tras un conflicto laboral con los centros de salud rurales en el que se reivindicaban mejoras en la organización y gestión de las urgencias, se creó un Servicio de Urgencias Rural (SUR) compuesto por personal sanitario específico para la realización de guardias en zona rural y no incluido en la estructura de los centros de salud ${ }^{26}$.

En la actualidad, la atención a la urgencia extrahospitalaria se realiza desde tres dispositivos: los servicios de urgencia ru- rales (SUR), los servicios normales de urgencia (SNU) que atienden a las poblaciones semiurbanas de Estella, Tafalla, Tudela y el Servicio de Urgencias Extrahospitalaria (SUE) que abarca Pamplona y comarca.

En los dos años de desarrollo de estos cambios organizativos han surgido críticas que cuestionan la precipitación de estas medidas, fruto de una presión laboral y no entroncadas en una adecuada planificación acorde con las necesidades de atención urgente. También se ha cuestionado la eficiencia de estas medidas y si han sido útiles para mejorar la calidad de la atención.

Estas razones han motivado la realización de este estudio con el objetivo de conocer la opinión de personas expertas sobre los problemas que tiene la atención de las urgencias extrahospitalarias en Navarra y proponer recomendaciones de mejora en base a dicha opinión.

\section{MATERIAL Y MÉTODOS}

Se realizó un estudio Delphi en el que se invitó inicialmente a 37 personas con amplia experiencia profesional en la atención de urgencias extrahospitalarias y hospitalarias, o con experiencia en gestión sanitaria. El grupo era heterogéneo en cuanto a formación y experiencia profesional. Lo componían: 9 médicos y 7 enfermeras de los servicios de urgencias extrahospitalarias; 15 médicos de familia de centro de salud; 6 médicos que realizan guardias de urgencias de hospital. Todos ellos contaban con más de 10 años de experiencia profesional relacionada con las urgencias y abarcan todas las áreas sanitarias en que se agrupa la población.

El grupo investigador contactó con cada participante para acordar su participación y posteriormente por medio del correo electrónico se les envió un primer cuestionario abierto, en el que se les solicitaba una valoración general de los problemas que detectaban en la organización de la atención a las urgencias y sus propuestas de solución. Tras el análisis del contenido de las respuestas, se elaboró un segundo cuestionario para aclarar aspectos en los que se detectaban desacuerdos en las ideas propuestas. 
En un tercer cuestionario con preguntas cerradas se identificaba el grado de acuerdo o desacuerdo con los problemas y propuestas de mejora que el grupo había identificado. En este tercer y último cuestionario, se solicitó a cada experto que, además, seleccionara los 15 problemas y 15 propuestas de solución más importantes, ordenándolas de mayor a menor importancia. Para el análisis de estas prioridades se asignó 15 puntos a la primera opción y de modo descendente a las siguientes.

Tanto los problemas como las propuestas de solución se cuantifican en formato de escala Likert.

Se consideran, únicamente, aquellos problemas y aquellas propuestas de solución en los que, al menos, el $50 \%$ de los expertos muestren su acuerdo y que su valor de prioridad supere el percentil 25. Realizamos un análisis de clúster jerárquico para identificar los grupos de problemas y propuestas en base a su nivel de prioridad.

\section{RESULTADOS}

$\mathrm{Al}$ primer cuestionario abierto respondieron 29 (76,3\%) expertos. En su análisis se pudieron identificar 213 ideas referentes a problemas en la atención de las urgencias y 150 propuestas de mejora.

Al segundo cuestionario respondieron 19 expertos $(51,4 \%)$ y en él se pedía su opinión acerca de 3 cuestiones donde las propuestas de solución estaban enfrentadas en 2 grandes grupos:

1. Demanda excesiva de urgencias: un grupo consideraba el abuso de la población como condicionante de un exceso de demanda, mientras que otro grupo señalaba la responsabilidad en la gestión de los servicios sanitarios como el determinante de esta situación.

2. Modelo de atención a las urgencias: un grupo señalaba que los servicios de urgencia deben atender todas las urgencias, incluso en horario de atención continuada y que el personal de atención primaria debe dedicarse a atender las consultas en centro y domicilios programados. Otro grupo, por contra, opina que la atención primaria debe atender todas las necesidades de la población y la atención continuada y las urgencias son parte de ella.

3. Uso indebido de la demanda de urgencias: un grupo opina a favor de penalizar el mal uso de la demanda. Otro grupo señala la necesidad de campañas de sensibilización y educación de la población en el uso adecuado de la urgencia.

Finalmente, al tercer cuestionario respondieron 31 expertos $(81,6 \%)$, de los cuales 11 (35,5\%) eran personal de urgencia extrahospitalaria. Se señalan un total de 42 problemas y 55 propuestas de solución.

\section{Identificación de problemas de las urgencias extrahospitalarias}

Del total de problemas señalados, 28 (66,7 \%) reunían los requisitos de selección.

El análisis de clúster permite visualizar 4 grupos: la demanda de urgencias por patología banal y el desconocimiento de las necesidades de atención se señalan como los 2 problemas prioritarios y se agrupan (Tabla 1) en un primer clúster con prioridad media de 275,0 y desviación estándar 31,1 . Los tres siguientes cluster muestran problemas con prioridades medias de 191,5 (2 ítems), 105,4 (12 ítems) y 63,1 (12 ítems).

Tabla 1. Análisis de clúster jerárquico de los problemas con un acuerdo $>50 \%$ y nivel prioridad superior al percentil 25

\begin{tabular}{c|c|c|c|c|c|c}
\hline Cluster & N=28 & Media & Desviación estándar & Mediana & Percentil 25 & Percentil 75 \\
\hline 1 & 2 & 275,0 & 31,1 & 275,0 & 253,0 & 297,0 \\
2 & 2 & 191,5 & 14,9 & 191,5 & 181,0 & 202,0 \\
3 & 12 & 105,4 & 14,9 & 103,5 & 96,5 & 110,0 \\
4 & 12 & 63,1 & 10,7 & 63,0 & 54,0 & 72,0 \\
\hline
\end{tabular}


La tabla 2 muestra los problemas ordenados por el análisis de clúster jerárquico con su nivel de prioridad y acuerdo alcanzado.

Tabla 2. Problemas identificados con más del $50 \%$ de acuerdo y prioridad percentil $>25$

\begin{tabular}{|c|c|c|c|c|}
\hline Orden & Descripción & Prioridad & \% Acuerdo & Clúster \\
\hline 1 & $\begin{array}{l}\text { La población utiliza los servicios de urgencia para patología banal, } \\
\text { como una consulta más }\end{array}$ & 297 & 93,5 & 1 \\
\hline 2 & $\begin{array}{l}\text { No se conocen con precisión las necesidades reales de atención a las } \\
\text { urgencias }\end{array}$ & 253 & 83,9 & 1 \\
\hline 3 & $\begin{array}{l}\text { Los SUR se adscriben a un centro de salud, pero no se integran en el } \\
\text { trabajo del EAP }\end{array}$ & 202 & 71 & 2 \\
\hline 4 & $\begin{array}{l}\text { No tenemos una definición operativa de lo que consideramos } \\
\text { urgencia a partir de la cual definir los servicios necesarios }\end{array}$ & 181 & 74,2 & 2 \\
\hline 5 & $\begin{array}{l}\text { Escasa utilización de las consultas de enfermería en la atención a las } \\
\text { urgencias con limitaciones para asumir una mayor responsabilidad. }\end{array}$ & 142 & 71 & 3 \\
\hline 6 & La población desconoce el concepto-significado de urgencia & 121 & 67,7 & 3 \\
\hline 7 & $\begin{array}{l}\text { El personal de urgencias no pertenece a ninguna Dirección/Servicio } \\
\text { específico }\end{array}$ & 114 & 64,5 & 3 \\
\hline 8 & $\begin{array}{l}\text { Existen ineficiencias por la infrautilización de muchos SUR, por muy } \\
\text { escasa demanda }\end{array}$ & 106 & 74,2 & 3 \\
\hline 9 & $\begin{array}{l}\text { Es un problema la existencia de un grupo de hiperfrecuentadores que } \\
\text { realizan consultas reiterativas por el mismo proceso }\end{array}$ & 104 & 74,2 & 3 \\
\hline 10 & $\begin{array}{l}\text { No se cuenta con la participación y experiencia de sociedades } \\
\text { científicas y del personal de urgencias en la planificación y gestión de } \\
\text { los servicios de urgencias }\end{array}$ & 104 & 77,4 & 3 \\
\hline 11 & $\begin{array}{l}\text { Los centros de salud utilizan el servicio de urgencias como una } \\
\text { consulta de "rebosamiento", para minimizar la presión asistencial } \\
\text { sobre el EAP }\end{array}$ & 103 & 54,8 & 3 \\
\hline 12 & Hay una formación deficiente en la atención de las urgencias vitales & 101 & 64,5 & 3 \\
\hline 13 & Faltan protocolos de actuación y unidad de criterios en SOS & 99 & 71 & 3 \\
\hline 14 & Ausencia de una dirección profesional de los servicios de urgencia & 94 & 76,7 & 3 \\
\hline 15 & $\begin{array}{l}\text { Utilización de coches propios para desplazamientos a avisos } \\
\text { urgentes, domicilios, etc. }\end{array}$ & 89 & 71 & 3 \\
\hline 16 & $\begin{array}{l}\text { Material para la atención a domicilio inadecuado y no normalizado, } \\
\text { muy pesado, de difícil transporte }\end{array}$ & 88 & 71 & 3 \\
\hline 17 & $\begin{array}{l}\text { Existe una escasa formación en la atención de urgencias de } \\
\text { problemas de salud mental }\end{array}$ & 81 & 77,4 & 4 \\
\hline 18 & Hay una ausencia de cultura de equipo en el personal de urgencias & 76 & 64,5 & 4 \\
\hline 19 & $\begin{array}{l}\text { La población a veces utiliza violencia gratuita, chantaje, para la } \\
\text { resolución de su demanda }\end{array}$ & 74 & 67,7 & 4 \\
\hline
\end{tabular}


IDENTIFICACIÓN DE PROBLEMAS Y PROPUESTAS PARA MEJORAR LAATENCIÓN DE LAS URGENCIAS...

\begin{tabular}{c|l|c|c|c}
\hline Orden & \multicolumn{1}{|c|}{ Descripción } & Prioridad & \% Acuerdo & Clúster \\
\hline 20 & Inexistencia de ropa y calzado adecuado a las condiciones de trabajo & 70 & 51,6 & 4 \\
\hline 21 & $\begin{array}{l}\text { El personal de urgencias tiene problemas con las libranzas tras la } \\
\text { guardia, problemas de sustitución en vacaciones, bajas.. }\end{array}$ & 67 & 54,8 & 4 \\
\hline 22 & $\begin{array}{l}\text { El personal de urgencias se siente solo desmotivado y con } \\
\text { insatisfacción vocacional }\end{array}$ & 66 & 54,8 & 4 \\
\hline 23 & $\begin{array}{l}\text { No hay protocolos de intervención en "urgencias sociales” orientados } \\
\text { a evitar su medicalización }\end{array}$ & 60 & 76,7 & 4 \\
\hline 24 & No está clara la cadena de mando en las urgencias & 55 & 58,1 & 4 \\
\hline 25 & $\begin{array}{l}\text { Son frecuentes los problemas de coordinación con urgencias } \\
\text { hospitalarias }\end{array}$ & 54 & 51,6 & 4 \\
\hline 26 & Hay déficit en formación para las urgencias pediátricas & 54 & 61,3 & 4 \\
\hline 27 & $\begin{array}{l}\text { La población desconoce la existencia de diferentes niveles } \\
\text { asistenciales donde poder acudir, de acuerdo a la gravedad de la } \\
\text { patología que presente }\end{array}$ & $\begin{array}{l}\text { Deficiente coordinación entre urgencias-SUE y urgencias de Salud } \\
\text { Mental }\end{array}$ & 50 & 64,5 \\
\hline
\end{tabular}

\section{Identificación de propuestas de solución}

Cumplieron los criterios de selección 40 propuestas que representan el $72,7 \%$ del total de propuestas señaladas en el tercer cuestionario. La tabla 3 muestra 4 grupos identificados mediante el análisis de clúster. Destaca la necesidad de diseñar un plan estratégico para la atención a urgencias que conforma un grupo claramente diferenciado del resto con un valor de 310 puntos de prioridad. El resto de clúster muestran los siguientes valores medios de prioridad: 162,6 (8 ítems); 106 (7 ítems) y 44,8 (24 ítems).

Tabla 3. Análisis de clúster jerárquico de las propuestas con un acuerdo $>50 \%$ y nivel prioridad superior al percentil 25

\begin{tabular}{c|c|c|c|c|c|c}
\hline Clúster & N=40 & Media & DS & Mediana & Percentil 25 & Percentil 75 \\
\hline 1 & 1 & 310,0 & & 310,0 & 310,0 & 310,0 \\
2 & 8 & 162,6 & 15,6 & 162,0 & 151,5 & 170,0 \\
3 & 7 & 106,0 & 17,3 & 105,5 & 91,0 & 121,0 \\
4 & 24 & 44,8 & 17,8 & 47,0 & 29,0 & 64,0 \\
\hline
\end{tabular}

La tabla 4 muestra las propuestas de solución con su nivel de prioridad y acuerdo. 
Tabla 4. Propuestas de solución con más del 50 \% de acuerdo y percentil de prioridad > 25

\begin{tabular}{|c|c|c|c|c|}
\hline Número & Descripción & Prioridad & Acuerdo & $\begin{array}{c}\text { Clúster } \\
\text { jerárquico } \\
4 \text { grupos }\end{array}$ \\
\hline 1 & $\begin{array}{l}\text { Es necesario diseñar un plan estratégico para la atención de las } \\
\text { urgencias en medio rural y urbano con una definición de recursos } \\
\text { acorde con las necesidades }\end{array}$ & 310 & 96,8 & 1 \\
\hline 2 & $\begin{array}{l}\text { Es necesario enviar mensajes claros a la población indicando que la } \\
\text { atención en los PAC es para urgencias y que no son una continuación } \\
\text { de las consultas normales de su centro de salud }\end{array}$ & 192 & 96,8 & 2 \\
\hline 3 & $\begin{array}{l}\text { Hay que integrar a los SUR como parte del EAP equiparando sus } \\
\text { derechos, integrando su trabajo en las funciones del EAP }\end{array}$ & 175 & 71,0 & 2 \\
\hline 4 & $\begin{array}{l}\text { El sistema sanitario debe establecer acciones sistemáticas de } \\
\text { información y educación a la población sobre el uso correcto de los } \\
\text { servicios }\end{array}$ & 165 & 93,5 & 2 \\
\hline 5 & $\begin{array}{l}\text { Habría que crear una mesa de profesionales para analizar necesidades } \\
\text { de las urgencias y recoger sus propuestas. }\end{array}$ & 162 & 90,3 & 2 \\
\hline 6 & $\begin{array}{l}\text { En los EAP es necesario establecer plantillas cerradas suficientes } \\
\text { para la atención normal y las urgencias, contemplando libranzas, } \\
\text { sustituciones, vacaciones, etc. }\end{array}$ & 162 & 74,2 & 2 \\
\hline 7 & $\begin{array}{l}\text { Hay que analizar las experiencias de otros países y otras comunidades } \\
\text { autónomas en la planificación de las urgencias. }\end{array}$ & 153 & 93,5 & 2 \\
\hline 8 & $\begin{array}{l}\text { La implantación de "Turnos Deslizantes" (un día a la semana los } \\
\text { cupos de mañana pasan por la tarde y viceversa) sería una buena } \\
\text { manera de mejorar la accesibilidad sin cargas de trabajo accesorias } \\
\text { para los equipos }\end{array}$ & 150 & 74,2 & 2 \\
\hline 9 & $\begin{array}{l}\text { Hay que eliminar Puntos de Atención Continuada (PAC) rurales } \\
\text { concentrando recursos en un número menor centros con mayores } \\
\text { recursos para tender urgencias en centro y domicilio }\end{array}$ & 142 & 67,7 & 2 \\
\hline 10 & La enfermería debe ser el primer contacto con el paciente en urgencias & 129 & 74,2 & 3 \\
\hline 11 & $\begin{array}{l}\text { Hay que desarrollar un plan de formación específica y práctica en } \\
\text { los problemas de salud mas relevantes y prevalentes, orientados a } \\
\text { aumentar la capacidad de resolución de los profesionales de los EAP } \\
\text { y servicios de urgencias extrahospitalarios }\end{array}$ & 121 & 96,8 & 3 \\
\hline 12 & $\begin{array}{l}\text { Es necesario mejorar la accesibilidad EAP en horarios de tarde para } \\
\text { disminuir algunas demandas de atención urgente }\end{array}$ & 110 & 74,2 & 3 \\
\hline 13 & $\begin{array}{l}\text { La atención primaria debe atender todas las necesidades de la } \\
\text { población tanto la atención normal como las urgencias }\end{array}$ & 101 & 54,8 & 3 \\
\hline 14 & $\begin{array}{l}\text { En algunas zonas alejadas o dispersas habrá que considerar } \\
\text { situaciones especiales y dotar de recursos para la urgencia aunque } \\
\text { por razones de número de urgencias esperadas o población no se } \\
\text { justifiquen }\end{array}$ & 91 & 93,5 & 3 \\
\hline 15 & $\begin{array}{l}\text { Se deben desarrollar medidas de educación a la población orientadas } \\
\text { a mejorar su autocuidado en cuadros agudos autolimitados y } \\
\text { reagudizaciones crónicas }\end{array}$ & 84 & 96,8 & 3 \\
\hline 16 & $\begin{array}{l}\text { Se debería introducir un ticket moderador para las urgencias no } \\
\text { justificadas }\end{array}$ & 76 & 61,3 & 4 \\
\hline 17 & $\begin{array}{l}\text { Hay que crear la figura del jefe guardia extrahospitalaria para que } \\
\text { coordine todos los PAC }\end{array}$ & 69 & 58,1 & 4 \\
\hline 18 & $\begin{array}{l}\text { Hay que mejorar la formación en las urgencias derivadas de problemas } \\
\text { de salud mental }\end{array}$ & 67 & 93,5 & 4 \\
\hline
\end{tabular}




\begin{tabular}{|c|c|c|c|c|}
\hline Número & Descripción & Prioridad & Acuerdo & \begin{tabular}{|c|} 
Clúster \\
jerárquico \\
4 grupos
\end{tabular} \\
\hline 19 & $\begin{array}{l}\text { Hay que crear la figura de médico coordinador hospitalario que sea } \\
\text { referente para consultas directas desde los centros de urgencias con } \\
\text { misiones de coordinación y apoyo }\end{array}$ & 66 & 71,0 & 4 \\
\hline 20 & Hay que mejorar la coordinación de los avisos por parte del 112 & 66 & 87,1 & 4 \\
\hline 21 & $\begin{array}{l}\text { Se debe hacer más presente el Servicio Navarro de Salud en la gestión } \\
\text { de SOS-Navarra }\end{array}$ & 65 & 74,2 & 4 \\
\hline 22 & $\begin{array}{l}\text { Tras la atención de una urgencia no justificada, se debe informar } \\
\text { al paciente de la forma adecuada de utilizar los servicios, y pautar } \\
\text { tratamiento hasta que pueda acudir a consulta con su médico (dar la } \\
\text { medicación en urgencias) }\end{array}$ & 64 & 77,4 & 4 \\
\hline 23 & $\begin{array}{l}\text { Habría que definir un horario de consulta normal y otro en el que } \\
\text { únicamente se atendieran las urgencias, de tal manera que las } \\
\text { demandas no justificadas en urgencias se deben remitir a la atención } \\
\text { normal tras valorar al paciente }\end{array}$ & 52 & 74,2 & 4 \\
\hline 24 & Son necesarios PAC para las urgencias pediátricas & 52 & 66,7 & 4 \\
\hline 25 & $\begin{array}{l}\text { Es necesario poner en marcha protocolos de actuación de las } \\
\text { principales patologías urgentes }\end{array}$ & 49 & 100 & 4 \\
\hline 26 & $\begin{array}{l}\text { Informar a los pacientes del coste de los servicios mediante facturas } \\
\text { en la sombra }\end{array}$ & 49 & 67,7 & 4 \\
\hline 27 & $\begin{array}{l}\text { Hay que mejorar la dotación de ropa y calzado adecuado al personal } \\
\text { que realiza las guardias }\end{array}$ & 48 & 70,0 & 4 \\
\hline 28 & Dotar de vehículos propios a los SUR y EAP & 47 & 61,3 & 4 \\
\hline 29 & $\begin{array}{l}\text { En Pamplona habrá que integrar las UVI-móviles en el futuro servicio } \\
\text { único de urgencias }\end{array}$ & 41 & 71,0 & 4 \\
\hline 30 & $\begin{array}{l}\text { Es necesario cerrar los puntos de urgencia los sábados por la mañana } \\
\text { en Pamplona y comarca, abriendo un número menor de centros con } \\
\text { mayores recursos para tender urgencias en centro y domicilio }\end{array}$ & 40 & 80,0 & 4 \\
\hline 31 & Hay que potenciar la telemedicina & 40 & 83,9 & 4 \\
\hline 32 & $\begin{array}{l}\text { Hay que mejorar la infraestructura de los centros de salud: consultas, } \\
\text { áreas descanso, etc. }\end{array}$ & 38 & 64,5 & 4 \\
\hline 33 & $\begin{array}{l}\text { Es necesario homogeneizar los medios técnicos y funcionamiento de } \\
\text { los distintos centros }\end{array}$ & 32 & 90,3 & 4 \\
\hline 34 & $\begin{array}{l}\text { Hay que diversificar los medios de trasporte según las características } \\
\text { de la urgencia }\end{array}$ & 29 & 87,1 & 4 \\
\hline 35 & $\begin{array}{l}\text { Hay que posibilitar pasantías formativas por servicios de urgencias y } \\
\text { servicios específicos }\end{array}$ & 26 & 83,9 & 4 \\
\hline 36 & $\begin{array}{l}\text { Siempre hay que asegurar la posibilidad de las libranzas tras la } \\
\text { guardia }\end{array}$ & 23 & 74,2 & 4 \\
\hline 37 & $\begin{array}{l}\text { Hay que hacer una definición de los puestos de trabajo en las urgencias } \\
\text { y quién toma las decisiones claves en la urgencia-emergencia }\end{array}$ & 23 & 87,1 & 4 \\
\hline 38 & Hay que dotar de GPS para los desplazamientos fuera del centro & 21 & 77,4 & 4 \\
\hline 39 & $\begin{array}{l}\text { Hay que mejorar la selección de pacientes que precisan traslado en } \\
\text { ambulancia }\end{array}$ & 19 & 71,0 & 4 \\
\hline 40 & $\begin{array}{l}\text { Hay que dotar de más tecnología (móvil, radio) para comunicación } \\
\text { con los hospitales de referencia en casos graves. }\end{array}$ & 19 & 83,9 & 4 \\
\hline
\end{tabular}


En la tabla 5 se señalan las 11 propuestas con un nivel de consenso superior al $90 \%$ agrupadas por un nuevo análisis de clúster. La propuesta primordial es la necesidad de diseñar un plan estratégico de atención de las urgencias extrahospitala- rias. El desarrollo de acciones educativas poblacionales sobre el uso de los servicios de urgencias, formativas de los sanitarios y la necesidad de involucrar a los sanitarios en la corresponsabilidad son manifiestos.

Tabla 5. Propuestas de solución con mas del 90 \% de acuerdo y percentil de prioridad > 25

\begin{tabular}{|c|c|c|c|}
\hline Descripción & Prioridad & Acuerdo & $\begin{array}{c}\text { Clúster } \\
\text { jerárquico } \\
3 \text { grupos } \\
\end{array}$ \\
\hline $\begin{array}{l}\text { Es necesario diseñar un plan estratégico para la atención de las } \\
\text { urgencias en medio rural y urbano con una definición de recursos acorde } \\
\text { con las necesidades }\end{array}$ & 310 & 96,8 & 1 \\
\hline $\begin{array}{l}\text { Es necesario enviar mensajes claros a la población indicando que la } \\
\text { atención en los PAC es para urgencias y que no son una continuación de } \\
\text { las consultas normales de su centro de salud }\end{array}$ & 192 & 96,8 & 2 \\
\hline $\begin{array}{l}\text { El sistema sanitario debe establecer acciones sistemáticas de } \\
\text { información y educación a la población sobre el uso correcto de los } \\
\text { servicios }\end{array}$ & 165 & 93,5 & 2 \\
\hline $\begin{array}{l}\text { Habría que crear una mesa de profesionales para analizar necesidades } \\
\text { de las urgencias y recoger sus propuestas }\end{array}$ & 162 & 90,3 & 2 \\
\hline $\begin{array}{l}\text { Hay que analizar las experiencias de otros países y otras comunidades } \\
\text { autónomas en la planificación de las urgencias. }\end{array}$ & 153 & 93,5 & 2 \\
\hline $\begin{array}{l}\text { Hay que desarrollar un plan de formación específica y práctica en los } \\
\text { problemas de salud más relevantes y prevalentes, orientados a aumentar } \\
\text { la capacidad de resolución de los profesionales de los EAP y servicios de } \\
\text { urgencias extrahospitalarios }\end{array}$ & 121 & 96,8 & 3 \\
\hline $\begin{array}{l}\text { En algunas zonas alejadas o dispersas habrá que considerar situaciones } \\
\text { especiales y dotar de recursos para la urgencia aunque por razones de } \\
\text { número de urgencias esperadas o población no se justifiquen }\end{array}$ & 91 & 93,5 & 3 \\
\hline $\begin{array}{l}\text { Se deben desarrollar medidas de educación a la población orientadas } \\
\text { a mejorar su autocuidado en cuadros agudos autolimitados y } \\
\text { reagudizaciones crónicas }\end{array}$ & 84 & 96,8 & 3 \\
\hline $\begin{array}{l}\text { Hay que mejorar la formación en las urgencias derivadas de problemas } \\
\text { de salud mental }\end{array}$ & 67 & 93,5 & 3 \\
\hline $\begin{array}{l}\text { Es necesario poner en marcha protocolos de actuación de las principales } \\
\text { patologías urgentes }\end{array}$ & 49 & 100 & 3 \\
\hline $\begin{array}{l}\text { Es necesario homogeneizar los medios técnicos y funcionamiento de los } \\
\text { distintos centros }\end{array}$ & 32 & 90,3 & 3 \\
\hline
\end{tabular}

La figura 1 muestra el diagrama de Pareto de las propuestas de mayor consenso ordenadas según su nivel de prioridad. Se observa que con las 5 primeras propuestas se recogen en torno al $70 \%$ de las prioridades señaladas por los expertos. 


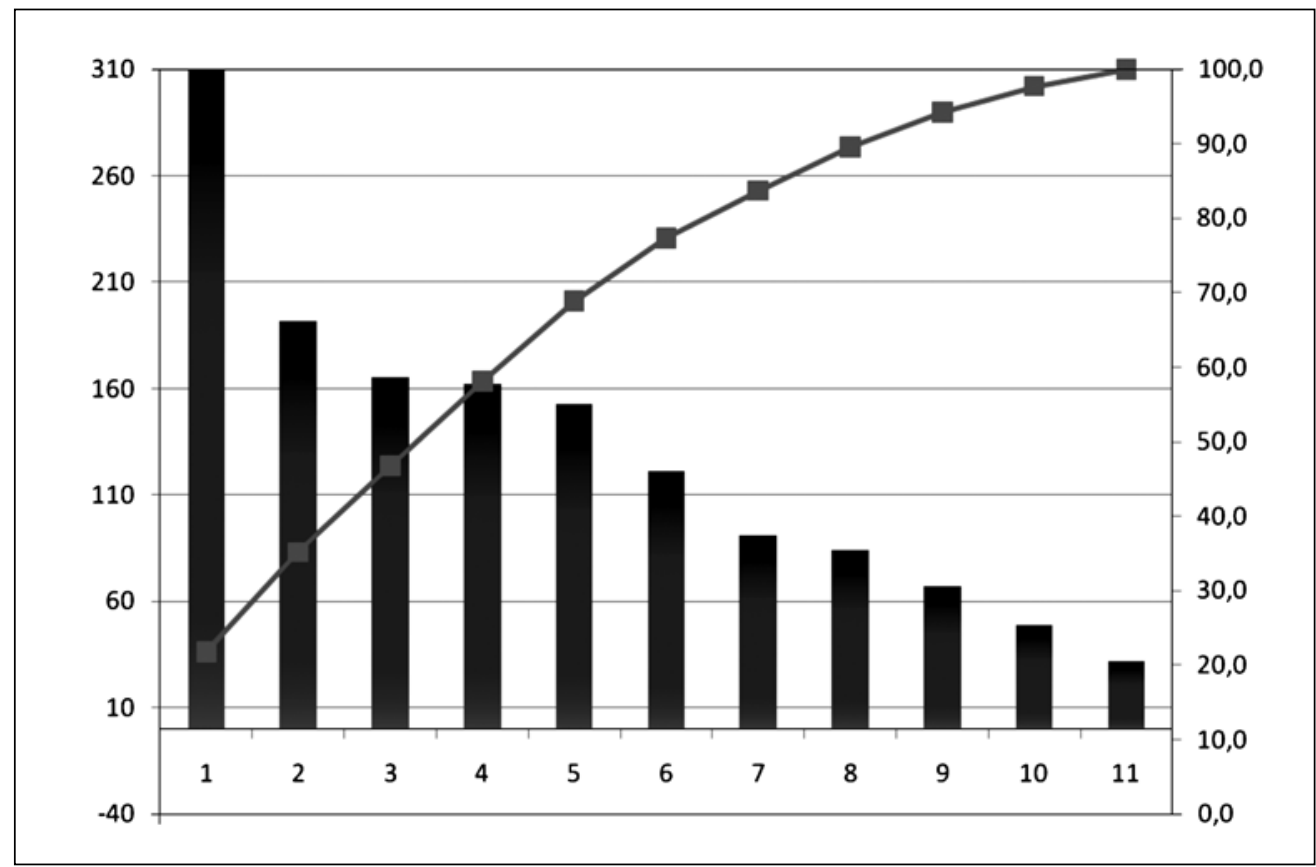

Fig. 1. Diagrama de Pareto con las propuestas de solución con más del 90 \% de acuerdo y percentil de prioridad $>25$.

\section{DISCUSIÓN}

El método cualitativo Delphi promueve una comunicación anónima, grupal y efectiva dirigida a la búsqueda de consenso, siendo útil en problemas poco definidos, complejos y multidimensionales como son los de índole social o sanitario ${ }^{27,28}$. Permite evitar sesgos debidos el efecto de los líderes del grupo sobre los demás evitando el pensamiento grupal.

Nuestro estudio, debido al número de expertos consultados, superior al mínimo recomendado ${ }^{27}$ y a su heterogeneidad, permite identificar perspectivas diferentes que aseguran una visión amplia del problema planteado.

Los autores definen al experto como aquel participante que puede realizar contribuciones válidas, dado que posee conocimientos y experiencia actualizada. Con esta premisa, el muestreo de los expertos es de conveniencia.

En nuestro estudio se realizan 3 rondas de intervención. No existe acuerdo en la bibliografía sobre el número de veces o rondas que se debe remitir el cuestionario, oscilando entre un mínimo de 2 y un máximo de 4 las rondas a realizar. Para los investigadores, el proceso termina cuando se considera que se ha llegado a un determinado nivel de consenso o que la información obtenida es suficiente y aclaratoria ${ }^{29}$.

El porcentaje de respuestas es alto $(83,8 \%)$ superior a estudios similares realizados en el ámbito sanitario ${ }^{29-31}$.

De los 28 problemas detectados, 5 están relacionados con la atención a la demanda; 3 sobre falta de integración de los SUR en los EAP; 3 sobre carencias de formación; 5 sobre déficit en derechos laborales; 3 sobre déficit en coordinación con otros servicios; 9 sobre déficit de planificación y dirección en los SUR.

Todos los eslabones de la cadena implicados en la urgencia están reflejados entre los problema señalados por los expertos.

Entre los puntos señalados, destaca un aspecto de responsabilidad privada, se- 
ñalado con diferente intensidad en varias publicaciones como es el hecho de que las urgencias se utilizan por los usuarios de manera indebida, para patología banal ${ }^{6-9,12}$, condicionados en parte por un déficit de educación sanitaria ${ }^{14-17}$, además de por una ausencia de corresponsabilidad. Este uso inadecuado es señalado por los expertos como de máxima prioridad entre los problemas planteados. Otros aspectos señalados en la literatura y manifestados por los expertos refieren el uso de los servicios por pacientes hiperfrecuentadores habituales $^{32,33}$ y la utilización de violencia por parte del usuario ${ }^{34-36}$, aspectos a considerar por los proveedores del servicio.

Se han señalado los efectos negativos del exceso de demanda de atención urgente extrahospitalaria no justificada, como son la demora en la atención y prolongación del dolor y sufrimiento de pacientes que no pueden ser atendidos con prontitud $^{7-9}$, insatisfacción de los pacientes y profesionales, descenso de la productividad, incremento de la frustración de los pacientes hacia los médicos, incremento de situaciones de queja y violencia ${ }^{2,37}$.

Los problemas planteados con origen o responsabilidad pública son numerosos, manifestándose incluso a nivel muy básico como es el desconocimiento de las necesidades reales de atención a las urgencias. Los déficit de integración entre los diferentes profesionales implicados como la no integración de los SUR en los equipos son señalados por los expertos, con la consiguiente despersonalización y pérdida de cultura de equipo, elemento clave en la atención sanitaria integral. Así mismo, se ha señalado el escaso papel que está desarrollando enfermería en la atención. Existe una creciente evidencia que enfermeras bien entrenadas pueden proporcionar servicios de gran calidad en atención primaria en colaboración con médicos y son caminos que se deberían explorar ${ }^{38,39}$. Igualmente se apunta a una inadecuada organización de los servicios disponibles para solventar las necesidades de los demandantes en coincidencia con otros autores $\mathbf{s}^{5,6,40}$.

La pasividad de la administración en cuanto a la necesidad de medios de trans- porte para atención a domicilio y el uso de prendas de ropa adecuadas e identificativas han estado ausentes en la planificación de recursos, con el consiguiente déficit en las condiciones laborales de los profesionales.

Los problemas de coordinación con otros servicios, especialmente con salud mental, urgencias hospitalarias y con el servicio de Coordinación-112 son manifiestos, por la gran dispersión de funciones y sumisión a los procedimientos de Protección Civil.

Se señala la necesidad de dimensionar correctamente la dotación médica de los SUR, tanto por exceso como por defecto, haciendo hincapié en las ineficiencias de algunos puntos de atención por la muy escasa demanda. Por último destacamos la preocupación por una falta de formación en áreas relevantes como son salud mental $\mathrm{y}$ urgencias vitales.

Se señalan un total de 40 ítems con propuestas de mejora identificadas por el grupo y agrupadas en las siguientes áreas: 2 ítems relacionados con mejoras en la planificación; 17 ítems con propuestas de mejora organizativas; 4 ítems sobre aspectos de coordinación; 6 ítems relacionados con aspectos educativos poblacionales; 6 ítems referidos a mejoras en la condiciones laborales; 3 ítems relacionados con mejorar la formación de los profesionales y 2 ítems de propuestas relacionadas con nuevas tecnologías.

El estudio avala un importante grupo de elementos de consenso. Entre las recomendaciones señaladas por los expertos se citan:

1. Necesidad de diseñar un plan estratégico de atención a la urgencia extrahospitalaria, con un análisis de necesidades y de los recursos necesarios.

La necesidad de sintonizar recursos con necesidades de atención es relevante en todo sistema de salud y esto nos lleva a cuestionar la actual distribución de los recursos de urgencias según las cuales se han equiparado la distribución espacial de los SUR a las actuales zonas de salud. Debe considerarse las necesidades de atención urgente como eje de la planificación de este servicio. 
El desarrollo de cambios organizativos dirigidos a facilitar el aumento del horario de atención del EAP mediante la implantación de turnos deslizantes son planteadas por los expertos.

Se deben incorporar aspectos de mejora de gestión y coordinación con otros servicios (bomberos, policía) y con el Centro de Coordinación de urgencias-112.

2. Necesidad de acciones de información y educación de la población. Deben ir dirigidas en varias direcciones, como es el autocuidado en cuadros limitados con implicación del propio paciente en el cuidado de su enfermedad y en el uso correcto de los servicios, tanto en frecuencia o intensidad de uso como en la utilización adecuada de los diferentes niveles de atención.

La implantación de triaje de enfermería y el posterior redireccionamiento de los pacientes con demandas no acordes a los servicios hospitalarios, hacia otros dispositivos más adecuados con la naturaleza de la demanda.

3. Necesidad de recabar la opinión de los sanitarios y analizar experiencias previas en territorios geográficos próximos que faciliten la correcta toma de decisiones en la planificación de la atención a las urgencias.

4. Necesidad de aumentar y potenciar los aspectos formativos de los profesionales fundamentalmente en la vertiente clínicoasistencial, y con especial referencia en el área de la salud mental, tanto en el aspecto sanitario como en el social y familiar. Esta formación debe ir dirigida a aumentar la capacidad de resolución de patología urgente y a un mejor conocimiento de los problemas de salud más prevalentes.

5. Necesidad de potenciar puntos de "especial actuación", con mayor dotación en recursos, con capacidad de respuesta adecuada e inmediata a la demanda de emergencias importantes.

Es necesario unificar PAC con muy escasa demanda en centros próximos, dotándolos de medios adecuados de transporte y material.

6. Necesidad de homogeneizar los medios técnicos de los distintos centros, así como integrar a los SUR con EAP.

\section{BIBLIOGRAFÍA}

1. Wai AK, Chor CM, Lee AT, Sittambunka Y, GraHAM CA, RAINER TH. Analysis of trends in emergency department attendances, hospital admissions and medical staffing in a Hong Kong university hospital: 5-year study. Int J Emerg Med 2009 2: 141-148.

2. DERLET RW. Overcrowding in emergency departments: increased demand and decreased capacity. Ann Emerg Med 2002; 39: 430432.

3. Lambe S, Washington DL, Fink A, Herbst K, Liu $\mathrm{H}$, ScuRA $\mathrm{J}$ et al. Trends in the use and capacity of California's emergency departments, 1990-1999. Ann Emerg Med 2002; 39: 389396.

4. SÁnchez J, Delgado AE, Muñoz H, Luna JD, JiméNEZ JJ, Bueno A. Frecuencia y características de la demanda atendida en un Servicio de Urgencia Hospitalario. Circuitos de atención. Emergencias. Emergencias 2005; 2: 52 61.

5. SÁnchez J, Bueno A. Factores asociados al uso inadecuado de un servicio de urgencias hospitalario. Emergencias 2005; 17: 138-144.

6. Antón MD, Peña JC, Santos R, Sempere E, MarTínez J y Férula LA. Demanda inadecuada a un servicio de urgencias pediátrico hospitalario: factores implicados. Med Clin (Barc) 1992; 99: 743-746.

7. Pérez-Ciordia I, Gullén Grima F. Urgencias hospitalarias y extrahospitalarias en Navarra. Razones que las motivan. An Sist Sanit Navar 2009; 32: 371-384.

8. Trzeciak S, Rivers EP. Emergency department overcrowding in the United States: an emerging threat to patient safety and public health. Emerg Med J 2003; 20: 402-405.

9. McNutt RA, Abrams R, Arons DC. Patient safety efforts should focus on medical errors. JAMA 2002; 287: 1997-2001.

10. Braun t, García L, Krafft T, Díaz-Regañón G. Frecuentación del servicio de urgencias y factores sociodemográficos. Gac Sanit 2002; $16: 139-144$.

11. Oterino de la Fuente D, Baños JF, Fernández V, RodRíguez-Álvarez A. Impacto en Asturias de las urgencias de atención primaria sobre las hospitalarias. Un análisis de cointegración de series temporales. Rev Esp Salud Pública 2007; 81: 191-200.

12. Brugos A, Guillén F, Mallor F, Fernández C. Modelos explicativos y predictivos de la carga asistencial médica: aplicación para el cálcu- 
lo del cupo máximo en medicina de familia que permita un mínimo de diez minutos por consulta. Aten Primaria 2003; 32: 23-29.

13. López N, Epelde F. Valoración del uso que hace la población inmigrante de un servicio de urgencias hospitalario. Emergencias 2010; 22: 109-112.

14. Anantharaman V. Impact of health care system interventions on emergency department utilization and overcrowding in Singapore Int J Emerg Med 2008; 1: 11-20.

15. Boyd M, Lasserson TJ, McKean MC, Gibson PG, Ducharme FM, HABY M. Intervenciones educativas para niños en posible visita al servicio de urgencias a causa del asma (Revisión Cochrane traducida). En: Biblioteca Cochrane Plus 2009 Número 3. Oxford: Update Software Ltd. Disponible en: http://www. update-software.com

16. TAPP S, LASSERSON TJ, Rowe BH. Intervenciones educativas para adultos que asisten al servicio de urgencias por asma aguda (Revisión Cochrane traducida). En: La Biblioteca Cochrane Plus, 2008 Número 4. Oxford: Update Software Ltd. Disponible en: http:// www.update-software.com.

17. Bhogal S, Zemek R, Ducharme FM. Planes de acción escritos para el asma en niños (Revisión Cochrane traducida). En: La Biblioteca Cochrane Plus, 2008 Número 4. Oxford: Update Software Ltd. Disponible en: http:// www.update-software.com/BCP/BCPGetDocument.asp?DocumentID=CD005306

18. Hsu J, Reed M, Brand R, Fireman B, Newhouse JP, SELby JV. Cost-sharing: patient knowledge and effects on seeking emergency department care. Med Care 2004; 42: 290-296.

19. WilKelmanN R. Co-payments for prescription drugs and the demand for doctor visits Evidence from a natural experiment. Health Econ 2004; 13: 1081-1089.

20. Miró O, Salgado E, Tomas S, Espinosa G, EstraDA C, MARTí C et al. Derivación sin visita desde los servicios de urgencia hospitalarios: cuantificación, riesgos y grado de satisfacción. Med Clin (Barc) 2006; 26: 8-93

21. Gómez-Jiménez J, Becerra O, Boneu F, Burgués L, PÀmies S. Análisis de la casuística de los pacientes derivables desde urgencias a atención primaria. Gac Sanit 2006; 20: 40-46.

22. Peiró S, Sempere Selva T, Oterino S. Efectividad de las intervenciones para reducir la utilización inapropiada de los servicios hospitalarios de urgencias. Revisando la literatura 10 años después del Informe del Defensor del Pueblo. Economía y Salud 1999; 33: 3-12.

23. OlshaKeR JS. Managing Emergency Department Overcrowding. Emerg Med Clin North Am 2009; 27: 593-603.

24. BABcock Irvin C, Wyer PC, Gerson LW. Preventive care in the emergency department, Part II: Clinical preventive services an emergency medicine evidence-based review. Society for Academic Emergency Medicine Public Health and Education Task Force Preventive Services Work Group. Acad Emerg Med 2000; 7: 1042-1054.

25. Busca P, Marrón R. La informatización en urgencias y emergencias. An Sist Sanit Navar 2010; 33 (Supl. 1): 69-76.

26. Servicio Navarro de Salud-Osasunbidea y centraLES SINDICALES. Acuerdo sobre atención continuada en Atención Primaria. 13 Marzo 2008.

27. Pineault R, Daveluy C. La Planificación Sanitaria: conceptos, métodos, estrategias. Ed Masson. Barcelona 1987.

28. Astigarraga E. El método Delphi. Universidad de Deusto San Sebastian. Disponible en: http://www.echalemojo.org/uploadsarchivos/metodo_delphi.pdf

29. YaÑez R, CuAdra R. La técnica Delphi y la investigación en los servicios de salud. Cienc Enferm 2008; 14: 9-15.

30. Marshall M, Lockwood A, Lewis, Fiander M. Essential elements of an early intervention service for psychosis: the opinions of expert clinicians. BMC Psychiatry 2004; 4: 17.

31. Brugos A, Arbeloa B, Astrain MV, López S, Otero M, Monreal C et al. Valoración del programa social de atención a personas con trastorno mental grave: un estudio Delphi. An Sist Sanit Navar 2007; 30: 101-112.

32. Riba D, Rodríguez-Rosich A, Gázquez M, Butı M. Pacientes hiperfrecuentadores en los servicios de urgencias. Estudio descriptivo en un hospital de segundo nível. Emergencias 2004; 16: 178-183.

33. Reid S, Wessely S, Crayford T, Hotopf M. Frequent attenders with medically unexplained symptoms: service use and costs in secondary care. Br J Psychiatry 2002; 180: 248-53.

34. KEnNEDY MP. Violence in emergency departments: under-reported, unconstrained, and unconscionable. Med J Aust 2005; 183: 362-365. 
35. Belayachi J, Berrechid K, Amlaiky F, Zekraoui A, ABOUQAL R. Violence toward physicians in emergency departments of Morocco: prevalence, predictive factors, and psychological impact. J Occup Med Toxicol 2010; 5: 27.

36. James A, Madeley R, Dove A. Violence and aggression in the emergency department. Emerg Med J 2006; 23: 431-434.

37. Moskop JC, Sklar DP, Geiderman JM, Schears RM, BooKman KJ. Emergency department crowding, part 1-concept, causes, and moral consequences. Ann Emerg Med 2009; 53 : 605-611.
38. Way D, Jones L, Baskerville B, Busing N. Primary health care services provided by nurse practitioners and family physicians in shared practice. CMAJ 2001; 165: 1210-1214.

39. Laurant M, Reeves D, Hermens R, Braspenning J, Grol R, SibBald B. Substitution of doctors by nurses in primary care. Disponible en: Cochrane Database Syst Rev 2005 Apr 18; CD001271

40. Aranaz JM, Martínez R, Gea MT, Rodrigo V, Antón P, Gómez F. ¿Por qué los pacientes utilizan los servicios de urgencias hospitalarios por iniciativa propia? Gac Sanit 2006; 20: 311-315. 